\title{
Pilot study and bioinformatics analysis of differentially expressed genes in adipose tissues of rats with excess dietary intake
}

\author{
JUN CHAO YUAN $^{1}$, THANESWARY YOGARAJAH ${ }^{2}$, SHERN KWOK LIM ${ }^{2}$, \\ GET BEE YVONNE TEE ${ }^{3}$ and BOON YIN KHOO ${ }^{2}$ \\ ${ }^{1}$ Department of Endocrinology, The First People's Hospital of Yunnan Province, Kunming, Yunnan 650032, P.R. China;
${ }^{2}$ Institute for Research in Molecular Medicine (INFORMM), Universiti Sains Malaysia, Penang 11800;
${ }^{3}$ School of Health Sciences, Universiti Sains Malaysia, Kubang Kerian, Kelantan 16150, Malaysia
}

Received March 24, 2019; Accepted December 11, 2019

DOI: $10.3892 / \mathrm{mmr} .2020 .11012$

\begin{abstract}
Excessive adipose tissue accumulation is an increasing health problem worldwide. The present study aimed to determine differentially expressed genes (DEGs) that are associated with the excessive accumulation of adipose tissues by PCR arrays in an excess dietary intake animal model. For this purpose, male Sprague Dawley rats were randomly assigned to 2 groups: Control (given an ordinary diet) and experimental (given twice the amount of the ordinary diet). After 2 months of feeding, the abdominal cavities of the rats from each group were opened, then subcutaneous and visceral adipose tissues were removed. The adipose tissues collected were then used for total RNA extraction and then reverse transcribed to cDNA, which was then used as a template to identify the DEGs of 84 transcripts for rat obesity by $\mathrm{RT}^{2}$ Profiler PCR Arrays. The results showed significant downregulation of bombesin-like receptor 3 (BRS3) and uncoupling protein 1 (UCP1) in visceral adipose tissues of experimental rats compared with those of the control rats, and differential gene expression analysis showed an association with fat cell differentiation and regulation of triglyceride sequestration, as well as fatty acid binding. The gene expression patterns observed in the present study, which may be associated with peroxisome proliferator-activated receptor- $\gamma$ (PPARG) on excessive visceral adipose tissue accumulation, may be useful in identifying a group of surrogate biomarkers for the early diet-induced accumulation of visceral adipose tissue detection in humans. The biomarkers can also be the specific targets for drug development to reduce excessive visceral adipose tissue accumulation in the body and its associated diseases.
\end{abstract}

Correspondence to: Dr Boon Yin Khoo, Institute for Research in Molecular Medicine (INFORMM), Universiti Sains Malaysia, H53 Jalan Inovasi, Penang 11800, Malaysia

E-mail: boonyin@usm.my

Key words: animal model, differentially expressed gene, excess dietary intake, excessive adipose tissue accumulation, PCR arrays, visceral adipose tissue

\section{Introduction}

Obesity, which is the accumulation of excessive adipose tissues, is a global health problem due to the potential for adverse effects on health, leading to various lethal diseases, including type 2 diabetes, high blood pressure, hypertension, dyslipidaemia, cardiovascular diseases and some types of cancer (1). The accumulated adipose tissue in the body can be classified into 2 categories: Subcutaneous tissue, which is stored under the skin, and visceral adipose tissue, which is stored in or around internal organs, such as the liver, blood vessels, kidney and pancreas (2-4). It is hypothesized that the accumulation of adipose tissue is age-related and visceral adipose tissue increases with age (5). However, more studies have demonstrated that obesity is mainly due to excess dietary intake (6-10). Visceral obesity, due to the storage of excess visceral adipose tissue, is more worrisome than subcutaneous obesity because the adipose tissue surrounds the vital organs and is metabolized by the liver, turning the tissue into blood cholesterol (11). In addition, visceral adipose tissue appears to be a key component that determines and predicts the development of a number of the aforementioned diseases (2). Moreover, breast cancer has been shown to exhibit complex associations with obesity (12).

Gene expression has been used to predict factors for being overweight, including gene fusion, co-occurrence and co-expression in the development of obesity (13). However, to the best of our knwoeldge, detection of adipose tissue accumulation based on gene expression profiles in subcutaneous and visceral adipose tissues by bioinformatics, which may lead to the identification of biomarkers to distinguish the type of adipose tissue accumulation, remains rare. Recent genetic studies have demonstrated that gene expression may play a role in the development of adipose tissue primarily by regulating the storage and release of energy from food (14-15). However, the negative regulation of adipogenic transcription factors and their downstream genes in visceral adipose tissue development remain to be elucidated. The negative regulation that causes visceral obesity has been demonstrated to reduce adipocyte proliferation and differentiation (16). As a result, the body does not have sufficient adipocytes to store extra fat; therefore, the fat is stored in internal organs during 
high-fat consumption (17). Increased expression of peroxisome proliferator-activated receptor- $\gamma$ (PPARG) has been detected in visceral adipose tissue (18). However, additional adipogenic-related genes need to be identified to understand the detailed underlying mechanism of visceral adipose tissue accumulation. The aim of the present study was to investigate differentially expressed genes (DEGs) at the mRNA level in the adipose tissues of an animal model fed with different amounts of food. The accumulated adipose tissues were then used for total RNA extraction and reverse transcribed to cDNA for the determination of differential expression of 84 genes using PCR arrays. Moroever, PCR arrays were used in the present study to measure the expression of various genes related to the excessive accumulation of adipose tissues in the body. The arrays are a reliable tool to analyze the expression of specific disease-related genes and biochemical pathways. PCR arrays comprise 96-well plates containing primers for a panel of genes involved in a specific biochemical pathway or disease, in addition to appropriate positive and negative controls. The technique combines the profiling capacities of a microarray with the performance of PCR and is becoming one of the standard approaches for measuring differential gene expression.

\section{Materials and methods}

Animal feeding and adipose tissue collection. Experiments involving the use of animals $(n=12)$ were performed according to the Guidelines For The Care And Use Of Animals For Scientific Purposes (19), after obtaining ethical approval from the Universiti Sains Malaysia Animal Ethics Committee [Permit no. USM/PPSF/50(085) Jld.2]. Male Sprague Dawley rats (weight, 80-100 g; age, 4 weeks) were obtained from the Animal House of Universiti Sains Malaysia. Rats were housed with three rats per cage at room temperature with a 12:12 h light:dark cycle and $50 \%$ relative humidity in the animal holding room. Only male rats were selected for the study as male rats are not susceptible to the physiological changes due to the oestrous cycle, the presence of which may have induced variations in the current study. All rats were given free access to drinking water and standard chow [Gold Coin Feedmills (Malaysia) Sdn. Bhd.]. After 2 weeks of acclimation to the diet and housing conditions, rats were randomly assigned to 1 of 2 groups: Control [given an ordinary diet, 15/100 g body weight per day $(n=3)$ ] or experimental [given twice the amount of the ordinary diet, 30/100 g body weight per day $(n=3)]$. Rats were provided with pre-weighed quantities of food each day and the quantity remaining in the food dish was weighed every $24 \mathrm{~h}$. The body weight of each rat in each group was recorded every week and the growth patterns were monitored prior to sacrifice via exposure to $100 \%$ carbon dioxide. A fill rate of $\sim 10 \%$ chamber volume/min was used. Death was confirmed based on a lack of respiration and faded eye colour. Then, the abdominal cavities of animals $(n=3)$ from each group were opened, whereby the subcutaneous and visceral adipose tissues were removed and weighed. All selection criteria of obese rats were performed based on a previous study (18). The collected adipose tissues were then used for total RNA extraction or rinsed in TRI Reagent ${ }^{\circledR}$ (Molecular Research Center), frozen in liquid nitrogen and stored at $-80^{\circ} \mathrm{C}$. The experiments were repeated twice ( 3 cohorts of 6 mice were used) to ensure reproducibility of the findings. A feeding period of 2 months was used because this period results in significant visceral adipose tissue accumulation in rats (18).

Total RNA extraction and cDNA synthesis. Total RNA extraction from subcutaneous and visceral adipose tissues was performed using TRI Reagent (Molecular Research Center), according to the manufacturer's protocols. The integrity of total extracted RNA was confirmed using $1 \%(\mathrm{w} / \mathrm{v})$ agarose gel electrophoresis, while the purity and the yield of the RNA were measured using a nanophotometer (Implen $\mathrm{GmbH})$. The integrity of total RNA was confirmed again by a 2100 Bioanalyzer (Agilent Technologies, Inc.) using the RNA 6000 Pico LabChip ${ }^{\circledR}$ Kit (Agilent Technologies, Inc.). Subsequently, $1 \mu \mathrm{g}$ of high-quality total RNA was reverse transcribed into cDNA using a RevertAid ${ }^{\mathrm{TM}}$ First Strand cDNA Synthesis Kit (Thermo Fisher Scientific, Inc.), according to the manufacturer's protocols. The success of cDNA synthesis was validated with conventional PCR amplification using rat GAPDH primers (forward 5'-CAAGTTCAACGGCAC AGTCAAG-3', reverse 5'-CTCCTGGAAGATGGTGATTGG T-3'). The PCR was performed using 2X PCR MasterMix (Thermo Fisher Scientific, Inc.) and SureCycler 8800 (Agilent Technologies, Inc.). For this, a volume of 2X PCR master mix (12.5 $\mu \mathrm{l}), 0.12 \mu \mathrm{M}$ of each forward and reverse primers, and $1.0 \mu \mathrm{l}$ of cDNA (100 ng) were mixed. A sufficient volume of deionized water $(9.5 \mu \mathrm{l})$ was added to the mixture, to bring the volume of the reaction solution to $25 \mu \mathrm{l}$ per vial. The program for the thermal cycler was carried out as follows: Initial denaturation at $95^{\circ} \mathrm{C}$ for $2 \mathrm{~min}$, followed by 35 cycles at $95^{\circ} \mathrm{C}$ for $30 \mathrm{sec}, 55^{\circ} \mathrm{C}$ for $30 \mathrm{sec}$ and $72^{\circ} \mathrm{C}$ for $1 \mathrm{~min}$ for annealing and the extension step.

PCR arrays. Following cDNA synthesis, DEGs were identified using $\mathrm{RT}^{2}$ Profiler $^{\mathrm{TM}}$ PCR Array Rat Obesity (cat. no. PAMM-017A-12; SABiosciences). Each plate for arrays contained 84 primer pairs that had been experimentally validated to ensure gene specificity and high amplification efficiency. Genes were categorized into 5 groups: i) Neuropeptides and receptors; ii) gut hormones and receptors; iii) adipocyte-derived peptides and receptors; iv) pancreas-derived peptides and receptors; and v) central nervous system-derived peptides and receptors. Reverse transcription-quantitative PCR (RT-qPCR) arrays were performed using an ABI 7000 Sequence Detection System (Applied Biosystems; Thermo Fisher Scientific, Inc.). For this purpose, reactions with a total volume of $25 \mu \mathrm{l}$, consisting of $\mathrm{RT}^{2}$ qPCR SYBR Green/Fluorescein, nuclease-free water and cDNA (template), were set up in each well of the plate. The reactions were then initiated at $95^{\circ} \mathrm{C}$ for $10 \mathrm{~min}$ to activate the HotStart polymerase, which was followed by 40 cycles of denaturation at $95^{\circ} \mathrm{C}$ for $15 \mathrm{sec}$ and primer annealing, as well as an extension at $60^{\circ} \mathrm{C}$ for $1 \mathrm{~min}$. A dissociation curve programme was added after the reactions were completed to generate the derivative dissociation curve of the reactions. The dissociation curve programme was initiated at $95^{\circ} \mathrm{C}$ for $1 \mathrm{~min}, 65^{\circ} \mathrm{C}$ for $2 \mathrm{~min}$ and $65^{\circ} \mathrm{C}$ to $95^{\circ} \mathrm{C}$ at $2^{\circ} \mathrm{C} / \mathrm{min}$. The intensity of each gene was then normalized to the intensity of housekeeping genes: $\beta$-actin (ACTB), $\beta$-2 microglobulin, GAPDH, heat shock protein $90 \alpha$ (cytosolic)-class B member 1 (HSP90AB1) or glucuronidase- $\beta$ (GUSB) (20). The normalized gene intensity of subcutaneous 
and visceral adipose tissues in control and experimental rats were then drawn as scatter plots using simple linear regression.

Clustering, functional enrichment and pathway analyses. The relationship between the normalized gene intensities of subcutaneous and visceral adipose tissues in control and experimental rats were then converted to fold change in a volcano plot generated using GraphPad Prism 7.05 (GraphPad Software, Inc.), whereby genes with a fold change $\geq 2$ and $\mathrm{P}<0.05$ were identified as statistically significant DEGs. A heatmap-dendrogram of $\log _{2}$ fold change for DEGs was then produced using Metascape (http://metascape.org/gp/index. html\#/main/step1). The heatmap showed upregulated genes (green), downregulated genes (red) and genes with no difference (black) in expression, whereas a dendrogram was constructed to show expression-based relationships across the entire set of expressed genes in the experiments. To further study the DEGs at a functional level, Gene Ontology (GO) functional enrichment analysis was performed using GeneMANIA (https://genemania.org/) (21-23). GeneMANIA discovered functionally similar genes using a wealth of genomics and proteomics data from a query gene. The algorithm of the modal was then weighted against each functional genomic dataset, according to its predictive value for the query, whereby a fold change discovery $\leq 0.05$ was set as the cut-off criterion for the enrichment analysis. The diagrams of network weight for BRS3 and UCP1 were plotted by $-\log _{10}$ ( $\Delta$ Weight). The list of genes likely to share function with the single query gene based on their interactions was listed.

Validation of selected genes. MCF-7 and MDA-MB-231 (American Type Culture Collection) were cultured with $6 \mathrm{ml}$ of $10 \%$ growth medium (DMEM; Gibco; Thermo Fisher Scientific, Inc.) supplemented with $10 \%$ (v/v) FBS (Gibco; Thermo Fisher Scientific, Inc.), $100 \mathrm{U} / \mathrm{ml}$ of penicillin and $100 \mathrm{mg} / \mathrm{ml}$ of streptomycin (Gibco; Thermo Fisher Scientific, Inc.) in a T-25 culture flask. The culture was incubated at $37^{\circ} \mathrm{C}$ in a humidified atmosphere of $5 \%(\mathrm{v} / \mathrm{v}) \mathrm{CO}_{2}$ until the growth of the cells reached approximately $70 \%$ confluence. After that, the old growth medium of the culture was discarded, the cells were trypsinized, and sub-cultured with fresh growth medium to 6-well plates. The cells were then ready to extraction of total RNA. Total RNA extraction of MCF-7 and MDA-MB-231 was performed using a RNeasy Mini kit (QIAGEN, Inc.). The concentration and integrity of the extracted RNA were verified, as described above. Extracted RNA of sufficient quality was reverse transcribed to cDNA using a RevertAid First Strand cDNA Synthesis kit (Thermo Fisher Scientific, Inc.). The product of reverse transcription (cDNA) was stored at $-20^{\circ} \mathrm{C}$ until it was used for the analysis of gene expression by conventional PCR. Gene-specific primers were designed for semi-quantitative amplification of the bombesin-like receptor 3 (BRS3), uncoupling protein 1 (UCP1) and GAPDH using Primer Express 3.0 software (Applied Biosystems; Thermo Fisher Scientific, Inc.). Forward human BRS3: 5'-GGCAGTTGTGAAGCCACT TGA-3', reserve human BRS3: 5'-AGACGCAGCCAGCTT TTACAC-3'; forward human UCP1: 5'-AGGACCAACGGC TTTCTTCAA-3', reserve human UCP1: 5'-CATAATGACGTT CCAGGATCCA-3'; forward human GAPDH: 5'-ACAGCC TCAAGATCATCAGCA-3', reverse human GAPDH: 5'-AGT
CTTCTGGGTGGCAGTGAT-3'. Semi-quantitative analysis is an analysis using a DNA ladder (marker) as an indication to measure the integrity of the PCR product. The gene expression was quantified using the ladder area; 'lower' and 'upper' ladders are internal standards used to align the ladder data with data from the sample wells. The area under the ladder was then compared with the sum of the sample peak areas. PCR was performed in a volume of $12.5 \mu \mathrm{l}$ of PCR Master Mix (Applied Biosystems; Thermo Fisher Scientific, Inc.), 20 pmol of each forward and reverse primer and $5 \mu \mathrm{l}$ of cDNA ( $25 \mathrm{ng}$ ). A sufficient volume of deionized water was added to the mixture to bring the volume of the reaction to $25 \mu 1$. The thermocycler program was carried out as follows: $50^{\circ} \mathrm{C}$ for $3 \mathrm{~min}$ and $95^{\circ} \mathrm{C}$ for $5 \mathrm{~min}$ as the short hot-start, followed by 35-40 cycles of $95^{\circ} \mathrm{C}$ for $10 \mathrm{sec}$ for denaturation, and annealing and extension steps at $60^{\circ} \mathrm{C}$ for $30 \mathrm{sec}$. The integrity of PCR products was confirmed by a 2100 Bioanalyzer (Agilent Technologies, Inc.) using a DNA 1000 LabChip $^{\circledR}$ Kit (Agilent Technologies, Inc.). Data analysis was carried out, according to the manufacturer's instructions, whereby the gene expression was quantified by comparing the integrity of a DNA ladder with the integrity from the sample. Each sample was analyzed in three replications. The experiment was performed and repeated at least twice.

The peak concentrations of PPARG, BRS3 and GAPDH were also analyzed in MK886-treated and control DMSO-treated MDA-MB-231 cells via PCR. The values were then converted to $\%$ of fold change for gene expression level analysis. To do this, MK886 (97\% purity; Sigma-Aldrich; Merck KGaA), which is PPARG inhibitor, was dissolved in DMSO (Bio Basic, Inc.) as a $10 \mathrm{mM}$ stock. The drug stock was stored at $-20^{\circ} \mathrm{C}$ and further diluted to the working concentration with assay medium [DMEM supplemented with $2 \%(\mathrm{v} / \mathrm{v})$ FBS and antibiotics]. Subsequently, $3 \mu \mathrm{M}$ of MK886 was added to the cells and the treated cells were incubated at $37^{\circ} \mathrm{C}$ in $5 \%(\mathrm{v} / \mathrm{v}) \mathrm{CO}_{2}$ for 2,4 and 6 days. The treatment criteria of MDA-MB-231 was performed based on the previous study (24). Following 2 days of treatment, the cells were harvested using a trypsin/EDTA solution (Gibco; Thermo Fisher Scientific, Inc.). MK886-treated and control DMSO-treated MDA-MB-231 cells were then subjected to total RNA extraction, cDNA conversion and peak concentration analysis by PCR and Bioanalyzer, as described above. The same method was used to calculate the target gene expression levels in MK886-treated and control DMSO-treated MDA-MB-231 cells following 4 and 6 days of treatment.

Data analysis. Graphs were extracted from GeneMANIA and all statistical analyses were performed using GraphPad Prism 7.05 (GraphPad Prism Software, Inc.). The significance of the data in scatter and volcano plots were analysed using unpaired t-tests. In contrast, two-way ANOVA with Tukey's multiple comparison post hoc test was used for the validation of the selected genes in cells. All values are expressed as mean $\pm \mathrm{SD}$. $\mathrm{P}<0.05$ was considered to indicate a statistically significant difference.

\section{Results}

Scatter plot analysis of normalized gene intensity. A total of 49 genes were detected from the analysis. The intensity 
$\mathrm{Aa}$
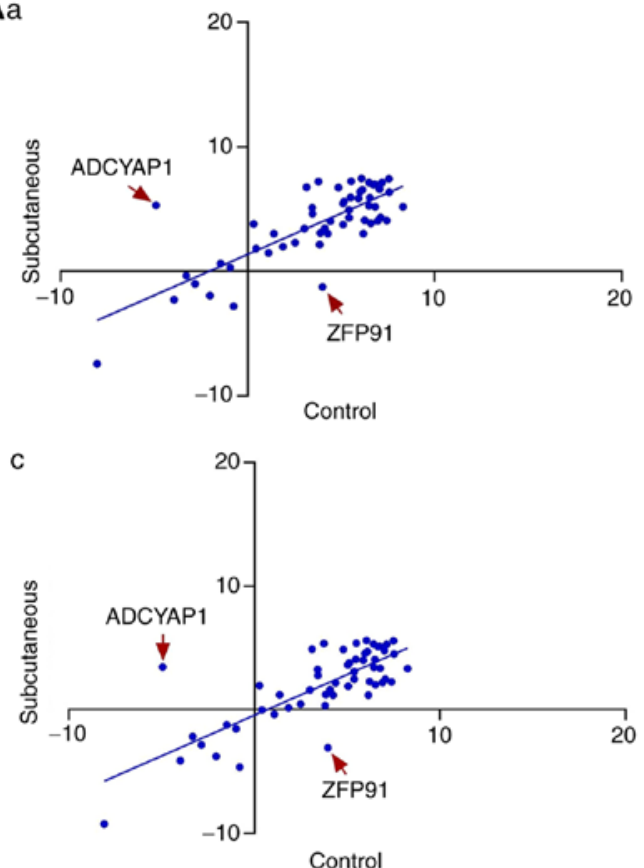

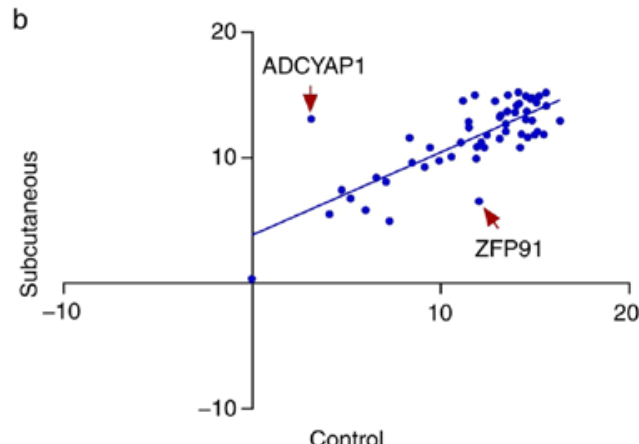

Control

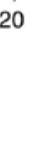

$\mathrm{B}$ a
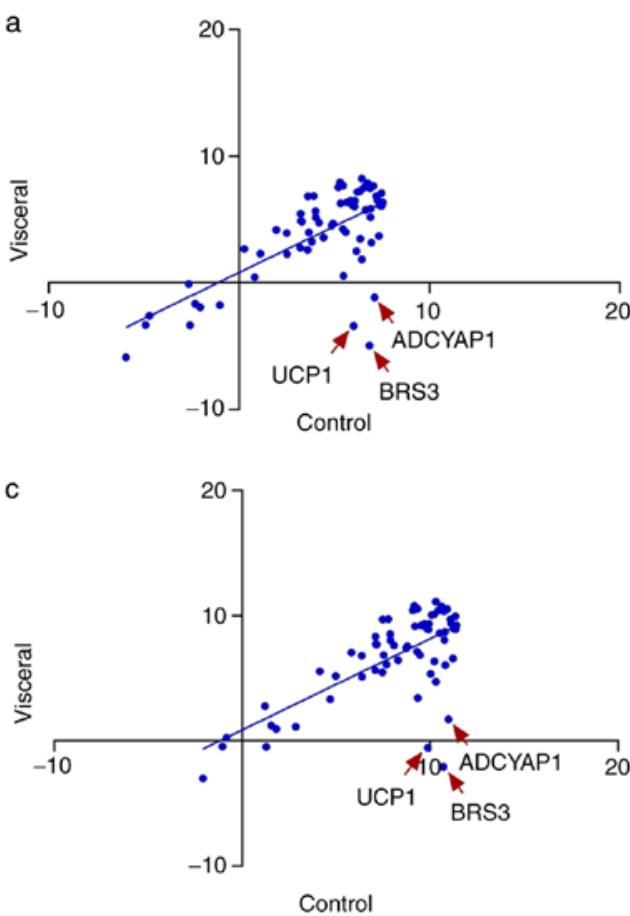

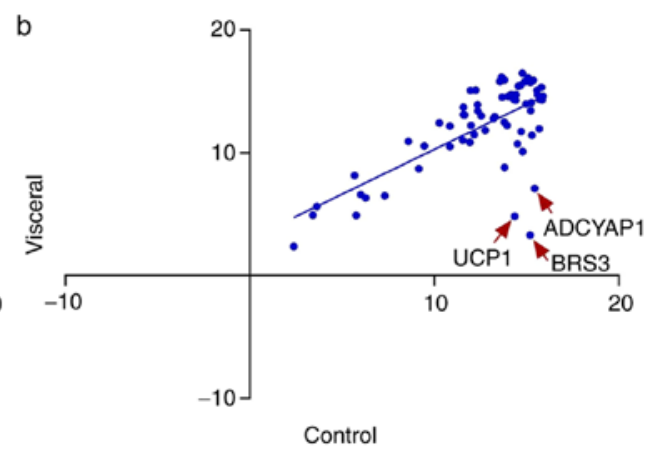

Control

normalized to plot of subcutaneous and visceral adipose tissue differentially expressed genes in control and experimental rats. (A) Gene profiles were $(\mathrm{Bb})$ GAPDH and $(\mathrm{Bc})$ HSP90AB1 in visceral adipose tissue. Red arrows indicate ADCYAP1 and ZFP91 as significantly upregulated and downregulated genes, respectively, in subcutaneous adipose tissue, whereas ADCYAP1, BRS3 and UCP1 were significantly downregulated in visceral adipose tissue. B2M, -2 microglobulin; HSP90AB1, heat shock protein $90 \alpha$ (cytosolic)-class B member 1; ADCYAP1, adenylate cyclase activating polypeptide 1; ZFP91, zinc finger protein 91; BRS3, bombesin-like receptor 3; UCP1, uncoupling protein 1.

of expression of each detected obesity related-gene in subcutaneous and visceral adipose tissues was normalized to various housekeeping genes provided by the arrays. Classic t-tests showed that adenylate cyclase activating polypeptide 1 (ADCYAP1; $\mathrm{P}<0.05$ ) and zinc finger protein 91 (ZFP91; $\mathrm{P}<0.05)$ were significantly upregulated and downregulated genes, respectively, in the subcutaneous adipose tissue of rats, which were overfed for 2 months (Fig. 1A), whereas ADCYAP1 $(\mathrm{P}<0.05), \mathrm{UCP} 1(\mathrm{P}<0.05)$ and BRS3 $(\mathrm{P}<0.05)$ were identified as significantly downregulated genes in visceral adipose tissues; however, no significantly upregulated genes were detected in this tissue between experimental and control rats (Fig. 1B). Statistical analysis identifying ADCYAP1, ZFP91, UCP1 and BRS3 as DEGs revealed that these genes 

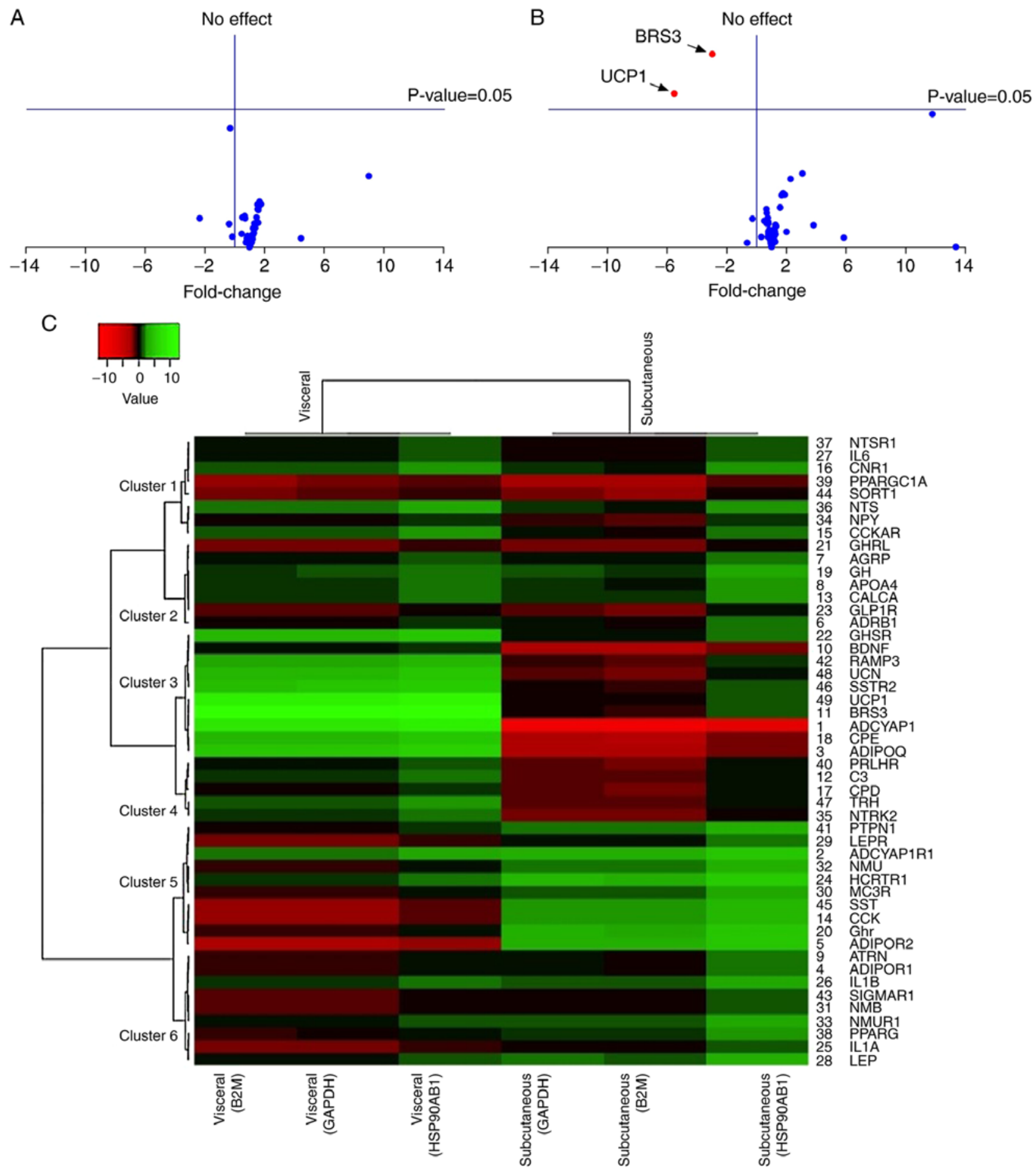

Figure 2. Clustering analyses of DEGs in adipose tissues in control and experimental rats. Analysis of volcano plots for (A) subcutaneous and (B) visceral adipose tissues, showing a fold change $\geq 2$ and a $\mathrm{P}<0.05$ cut-off for statistically significant DEGs. (C) Heatmap/dendrogram. The expression of each gene was normalized to the expression of the housekeeping genes B2M, GAPDH and HSP90AB1. Data are presented as fold change in volcano plot and $\log _{2}$ fold change in heatmap to reduce the differences in gene expression. Genes with higher expression levels are depicted in red, whereas genes with lower expression levels are depicted in green. Genes with no differences are depicted in black. DEGs, differentially expressed genes; BRS3, bombesin-like receptor 3; UCP1, uncoupling protein 1; B2M, $\beta$-2 microglobulin; HSP90AB1, heat shock protein $90 \alpha$ (cytosolic)-class B member 1.

are potentially associated with adipose tissue accumulation induced by excessive dietary intake in rats. In addition, the distribution of data within the plots is presented, where only the data normalized to GAPDH are distributed at the centre of the plots. This phenomenon probably indicated that GAPDH is a suitable housekeeping gene for the validation of gene expression in the present study using rats. The housekeeping genes ACTB and GUSB were also measured in the assays but were not detected in the present study.

Volcano plot and heatmap-dendrogram analyses for DEG identification. In using volcano plots to illustrate fold change, no gene was identified as significantly different in expression in the subcutaneous adipose tissue (Fig. 2A), whereas 2 genes, BRS3 and 
Table I. Selected differentially expressed genes in visceral adipose tissue.

\begin{tabular}{lccc}
\hline Gene & $\begin{array}{c}\text { Average expression } \\
\text { value }\left(\log _{2} \text { fold change }\right)\end{array}$ & P-value & Expression alteration \\
\hline BRS3 & -2.977 & 0.014996 & Downregulated \\
UCP1 & -5.527 & 0.035315 & Downregulated \\
\hline
\end{tabular}

BRS3, bombesin-like receptor 3; UCP1, uncoupling protein 1.

\section{A} a아
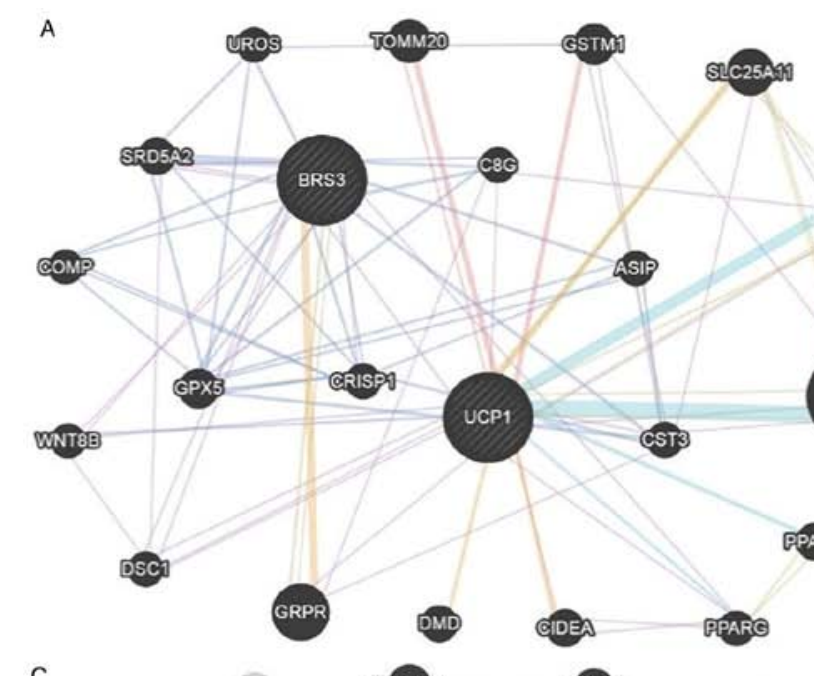

c

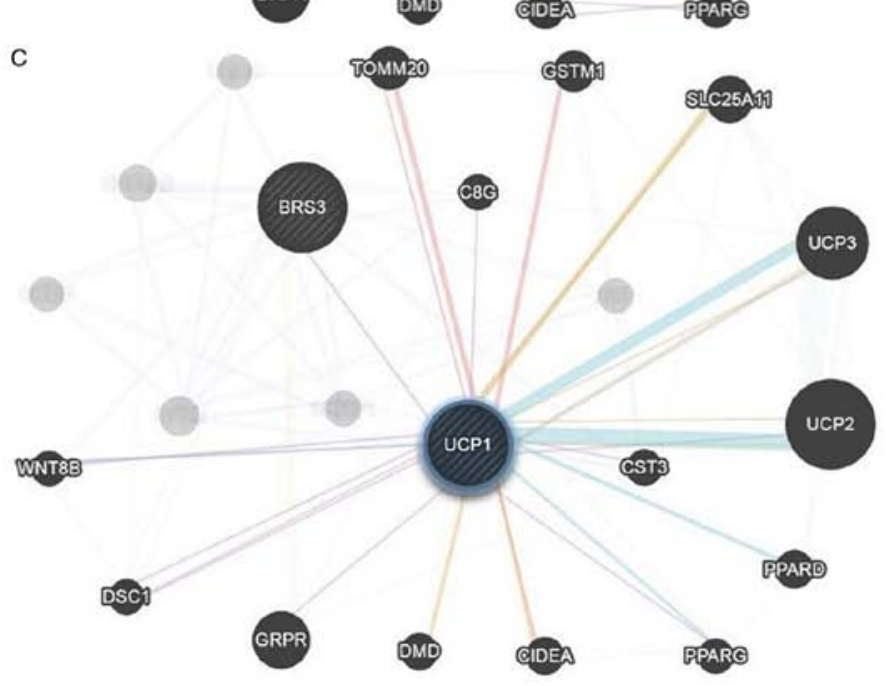

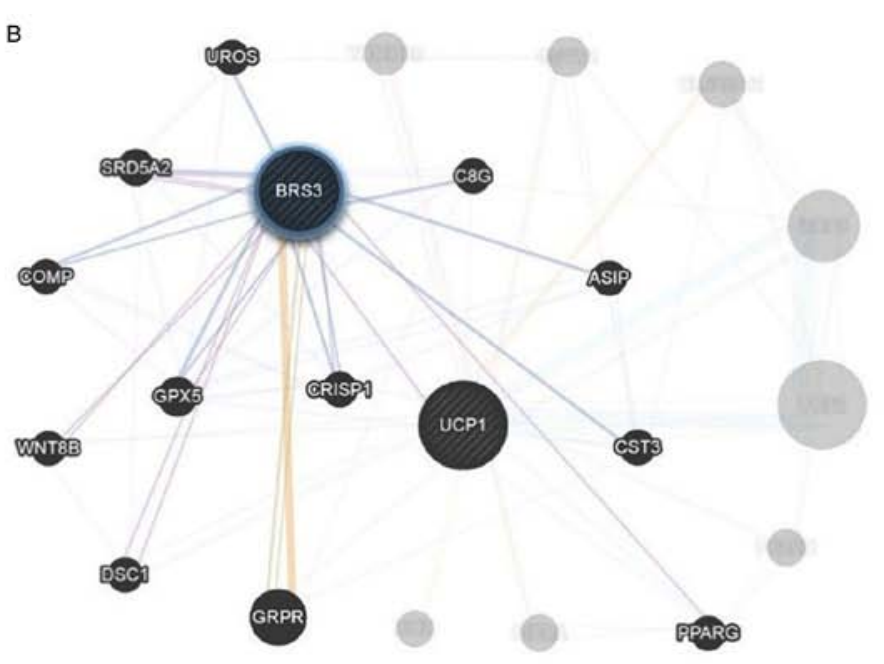

Figure 3. Composite networks for BRS3 and UCP1. Related genes are shown in circles for (A) BRS3 and UCP1, (B) BRS3 only and (C) UCP1 only. The interrelatedness of genes is reflected by the size of the circle. BRS3, bombesin-like receptor 3; UCP1, uncoupling protein 1.

UCP1, were identified as significantly downregulated in visceral adipose tissue (Fig. 2B), with fold changes of $12.0(\mathrm{P}<0.05)$ and $9.64(\mathrm{P}<0.05)$, respectively, using classic t-tests. The present study next explored obesity-related DEGs by clustering the $\log _{2}$ fold change for each gene in subcutaneous and visceral adipose tissues in a heatmap-dendrogram. Out of 49 analyzed genes in the heatmap, only 5 genes $(10.2 \%)$ were $\geq 2$-fold downregulated and 6 genes $(12.2 \%)$ were $\geq 2$-fold upregulated in subcutaneous tissue. In addition, 4 genes $(8.2 \%)$ showed $\geq 2$-fold downregulation and 9 genes $(18.4 \%)$ showed $\geq 2$-fold upregulation in visceral adipose tissue after removing the group of genes that were normalized to HSP90AB1 and shown to be incompatible (Fig. 2C). Based on $\mathrm{P}<0.05$ as the cut-off point, 8 genes, including brain-derived neurotrophic factor, BRS3, growth hormone receptor, growth hormone secretagogue receptor (GHSR), hypocretin receptor 1, somatostatin receptor 2 (SSTR2), urocortin (UCN) and UCP1, were identified as differentially expressed in visceral adipose tissue. The expression of BRS3 and UCP1 was also observed to significantly differ in visceral adipose tissue in both analyses. Therefore, BRS3 and UCP1 in visceral adipose tissue were selected for subsequent studies (Table I). A dendrogram that was constructed to show the expression-based relationship of BRS3 and UCP1 across the entire set of expressed genes in the experiment included GHSR, receptor activity-modifying protein 3, UCN, SSTR2, ADCYAP1, carboxypeptidase E and adiponectin (Cluster 3; Fig. 2C). 
Table II. Gene Ontology functional annotation of composite networks for BRS3 and UCP1.

\begin{tabular}{|c|c|c|c|}
\hline Function & Genes in network & Gene symbol & Fold change discovery \\
\hline \multirow[t]{2}{*}{ Fat cell differentiation } & 2 & PPARD & 0.0007 \\
\hline & & PPARG & 0.0005 \\
\hline \multirow[t]{2}{*}{ Sequestering of triglyceride } & 1 & CIDEA & 0.0007 \\
\hline & & PPARG & 0.0005 \\
\hline \multirow[t]{2}{*}{ Proton transport } & 2 & UCP2 & 0.0049 \\
\hline & & UCP3 & 0.0035 \\
\hline \multirow[t]{3}{*}{ Organelle inner membrane } & 3 & UCP2 & 0.0049 \\
\hline & & UCP3 & 0.0035 \\
\hline & & SLC25A11 & 0.0014 \\
\hline \multirow[t]{2}{*}{ Hydrogen transport } & 2 & UCP2 & 0.0049 \\
\hline & & UCP3 & 0.0035 \\
\hline \multirow[t]{4}{*}{ Mitochondrial membrane } & 4 & $\mathrm{UCP} 2$ & 0.0049 \\
\hline & & UCP3 & 0.0035 \\
\hline & & SLC25A11 & 0.0014 \\
\hline & & TOMM20 & 0.0011 \\
\hline \multirow[t]{3}{*}{ Mitochondrial inner membrane } & 3 & UCP2 & 0.0049 \\
\hline & & UCP3 & 0.0035 \\
\hline & & SLC25A11 & 0.0014 \\
\hline \multirow[t]{2}{*}{ Regulation of sequestering of triglyceride } & 1 & CIDEA & 0.0007 \\
\hline & & PPARG & 0.0005 \\
\hline \multirow[t]{2}{*}{ Electron transport chain } & 2 & UCP2 & 0.0049 \\
\hline & & UCP3 & 0.0035 \\
\hline \multirow[t]{2}{*}{ Fatty acid binding } & 1 & PPARD & 0.0007 \\
\hline & & PPARG & 0.0005 \\
\hline
\end{tabular}

GRPR, GSTM1, GPX5, SRD5A2, DMD, CST3, C8G, CRISP1, DSC1, ASIP, UROS, COMP and WNT8B have not yet been ascertained in the composite network. BRS3, bombesin-like receptor 3; UCP, uncoupling protein; PPAR, peroxisome proliferator-activated receptor; SLC25A11, mitochondrial 2-oxoglutarate/malate carrier protein.

Functional enrichment analysis of key DEGs in visceral adipose tissue. Construction of weighted composite gene-gene functional interaction networks for BRS3 and/or UCP1 from a list of genes using GeneMANIA are shown in Fig. 3. The networks included 20 gene interactions, in which 13 and 15 genes were associated with BRS3 and UCP1, respectively. The results of the list indicated that the key DEGs, BRS3 and UCP1, which are commonly found in visceral adipose tissue, are mostly associated with complement $\mathrm{C} 8 \gamma(\mathrm{C} 8 \mathrm{G})$, cystatin 3 (CST3), Wnt-8b (WNT8B), desmocollin-1 (DSC1), gastrin-releasing peptide receptor (GRPR) and PPARG, and that the functional annotations of each gene from GO are highlighted in Table II. PPARG may be closely associated with BRS3 and UCP1 in fat cell differentiation, sequestering of triglycerides and regulation of sequestering of triglycerides, and fatty acid binding in visceral adipose tissue development. In addition to PPARG, other genes, including C8G, CST3, WNT8B, DSC1 and GRPR, were also identified in the composite networks. The results also showed that BRS3 is associated with PPARG and WNT8B (Fig. 4A), whereas UCP1 is associated with PPARG, CST3, DSC1, C8G and GRPR (Fig. 4B). Therefore, BRS3, UCP1 and PPARG may be useful as biomarkers for the design of drugs or cost-effective agents to reduce visceral adipose accumulation and its associated diseases, including breast cancer.

Validation of BRS3 and UCP1 peak concentrations in breast cancer cell lines. The peak concentrations of BRS3 and UCP1 were analyzed in breast cancer cell lines using PCR and Bioanalyzer. The gene validation showed that the peak concentrations for both BRS3 and UCP1 were not detected in MCF-7 cells, whereby only GAPDH was identified as a detectable peak at $0.62 \mathrm{ng} / \mu \mathrm{l}$ (Table III). UCP1 was also not detected in MDA-MB-231 cells. Only BRS3 (3.72 ng/ $\mu \mathrm{l}$ ) and GAPDH $(0.36 \mathrm{ng} / \mu \mathrm{l})$ were detected in the cells, indicating that BRS3 may play an important role in the activity of highly metastatic breast cancer cells. A similar mRNA expression profile for PPARG and BRS3 was also observed when MDA-MB-231 cells were treated with MK886 (a PPARG inhibitor). MK886 (3 $\mu \mathrm{M})$ inhibited the level of PPARG mRNA expression by $\sim 80.15 \%$ of fold change $(\mathrm{P}<0.05)$ compared with control cells as early as 2 days following treatment (Fig. 5). The treatment was found to induce upregulation of PPARG mRNA expression $(121.55 \%$ of fold change, $\mathrm{P}<0.05)$ at day 4 of treatment compared to the control. Moreover, the mRNA expression level of PPARG was inhibited at day 6 following 
A

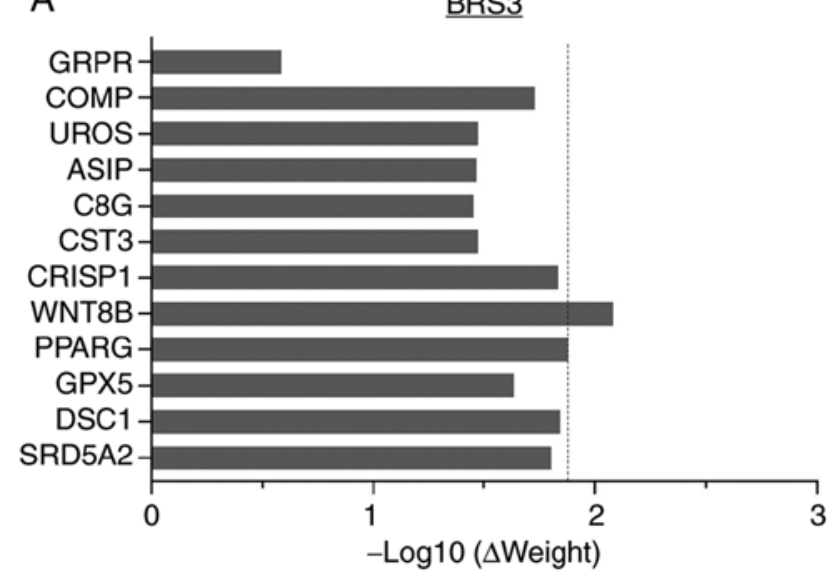

B

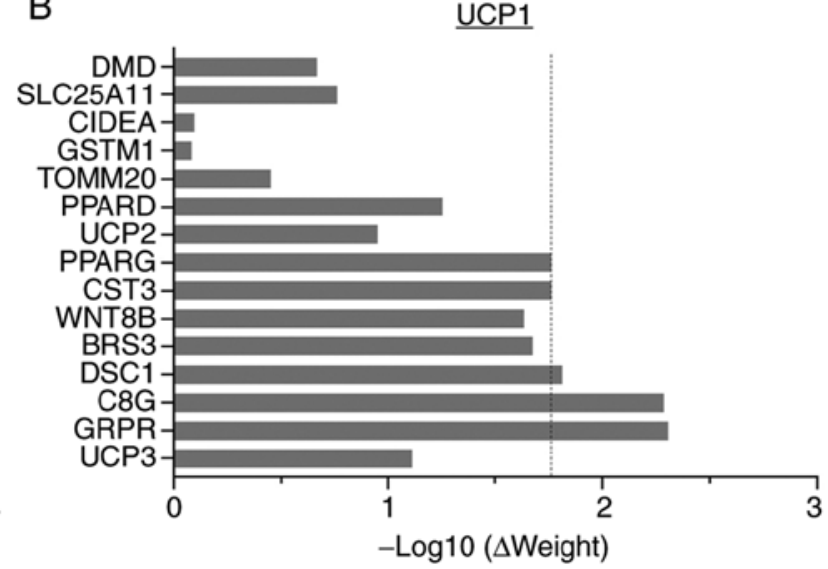

Figure 4. Functional roles of the key differentially expressed genes in visceral adipose tissue. (A) BRS3 and (B) UCP1. Network weight reflects the data source relevance for predicting the function of interest. BRS3, bombesin-like receptor 3; UCP1, uncoupling protein 1 .

Table III. Peak level of gene expression in breast cancer cells.

\begin{tabular}{llc}
\hline Cell line & Gene & Peak concentration $(\mathrm{ng} / \mu \mathrm{l})$ \\
\hline MCF-7 & BRS3 & Not determined \\
& UCP1 & Not determined \\
MDA-MB-231 & GAPDH & 0.62 \\
& BRS3 & 3.72 \\
& UCP1 & Not determined \\
& GAPDH & 0.36 \\
\hline
\end{tabular}

BRS3, bombesin-like receptor 3; UCP1, uncoupling protein 1.

treatment with MK886 ( 78.15\% of fold change) compared with the control, indicating that the drug effectively inhibited PPARG in MDA-MB-231 cells. There was also a significant difference between the mRNA expression levels of reduction in PPARG at day 6 compared with day 4 in MK886-treated MDA-MB-231 cells $(\mathrm{P}<0.05)$. Furthermore, a different expression profile of BRS3 in MDA-MB-231 cells was observed after treatement with the PPARG inhibitor for 6 days. A higher level of BRS3 expression was found when the PPARG inhibitor was first introduced to MDA-MB-231 cells for 2 days $(\sim 157.51 \%$ of fold change; $\mathrm{P}<0.05)$. In addition, BRS3 expression level was reduced to $\sim 79.74 \%$ of fold change $(\mathrm{P}<0.01)$ after 4 days, at which point PPARG expression was induced. The expression level of BRS3 was further increased to $\sim 128.10 \%$ of fold change at day 6 , when PPARG expression was downregulated. There was also a significant difference between the levels of BRS3 mRNA expression at day 6 compared with day 4 in MK886-treated MDA-MB-231 cells $(\mathrm{P}<0.05)$. This phenomenon further indicated that PPARG and BRS3 expression may be associated, which may play a role in the activity of highly metastatic breast cancer cells.

\section{Discussion}

The present study identified BRS3 and UCP1 as DEGs in visceral adipose tissue of rats with an excess dietary intake. The gene

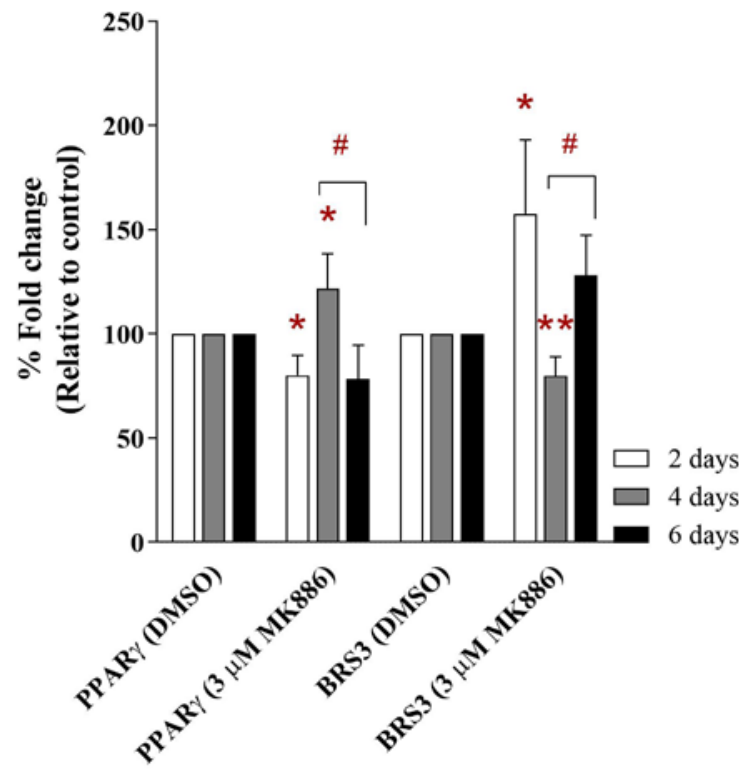

Figure 5. mRNA expression of PPARG and BRS3 in MDA-MB-231 cells. The cells were treated with $3 \mu \mathrm{M}$ of MK886 for 2, 4 and 6 days. DMSO was used as a diluent control for the present study. Data are shown as the mean \pm standard deviation of triplicate cultures. ${ }^{*} \mathrm{P}<0.05,{ }^{* *} \mathrm{P}<0.01$ vs. DMSO at the equivalent time point. ${ }^{\prime \prime} \mathrm{P}<0.05 \mathrm{vs}$. MK866 treatment group at different time points. PPARG, peroxisome proliferator-activated receptor- $\gamma$; BRS3, bombesin-like receptor 3 .

network may be associated with PPARG, as increased expression of PPARG was detected in visceral adipose tissue in our previous study (18). PPARG, which is widely described as playing an important role in adipocyte proliferation and differentiation, as well as in the conversion of various cells into adipose-like cells (25-30), was implicated in the visceral adipose tissue accumulation of the experimental rats in the present study. PPARG expression is also potentially associated with serum levels of $\mathrm{C}-\mathrm{C}$ motif chemokine ligand 2 and interleukin-6 in rats (18), which may facilitate the accumulation of visceral adipose tissue, indicating that PPARG serves a role in this phenomenon. However, whether PPARG is directly or inversely associated with BRS3 and UCP1 in visceral adipose tissue accumulation needs further investigation. 
The results of the present study suggested that BRS3, UCP1 and PPARG could be an important group of genes involved in the accumulation of excess visceral adipose tissue. BRS3 is an orphan $\mathrm{G}$ protein-coupled receptor that has been shown to regulate blood pressure and heart rate through a central sympathetic mechanism (31), whereas UCP1 is a primary regulator of thermogenesis that is likely to play an important role in the regulation of body adiposity (32). UCP1 has been demonstrated to be regulated by PPARG during adipocyte differentiation and lipid oxidation (33). However, studies into the association between BRS3 and PPARG remain rare. BRS3 is an important factor that should be considered in the gene network, as depletion of this gene alters adiposity, but also influences blood pressure and heart rate in animal models, which is undesirable in clinical treatment (31). The effects of social isolation on body weight gain, food consumption and responsiveness to a novel, as well as social, environment has been assessed in BRS3-deficient mice (34). However, the effect of BRS3 on visceral adiposity has yet to be fully elucidated. Notably, the expression of this gene was detected in highly metastatic breast cancer cells in the present study, which is consistent with a previous study describing that excessive accumulation of adipose tissues is one of the well-known risk factors for breast cancer (35). The present study also found that PPARG and BRS3 are associated. The strategy of PPARG inhibition must be considered with caution during anti-disease agent design, as inhibition of PPARG alone may not play an absolute role in body adiposity or disease reduction, but it may affect blood pressure and heart rate due to its association with BRS3. Therefore, BRS3 gene expression will be one of the essential targets in the gene network in future studies.

Furthermore, a lack of UCP1 may convey a tendency towards fat accumulation, which can be further confirmed by the analysis in the present study. The study showed that downregulation of UCP1 increased visceral adipose tissue accumulation. UCP1 depletion also results in poor thermoregulation and susceptibility to cold (32). As such, it is hypothesized that UCP1 may be an important factor in the development of anti-disease agents from local natural products in the future, whereby activation or upregulation of UCP1 is likely to regulate adiposity negatively. This is supported by the observation that reconstitution of UCP1 in the white adipose tissue of an animal model was demonstrated to decrease fat deposition and improve thermogenic capacity, which may also be a potential and valuable resource for the production of lean meat (32). All factors in this gene network require careful validation at cell-specific level before more costly tissue-specific validation is conducted to support their use in the agricultural sector or for potential anti-disease agent development.

The accumulation of adipose tissue is age-related (5). A previous report determined that a metabolic profile predictive of cardiovascular disease showed an increased risk in middle-aged premenopausal women compared with young women. Similarly, this phenomenon can also be explained by our previous study, which demonstrated a significant increase in the body weight of male rats as early as 2 months after the feeding regimen had begun, and which is probably due to the excessive accumulation of subcutaneous adipose tissue before visceral adipose tissue accumulation (18). Moreover, middle-aged individuals have been found to have elevated levels of total visceral adipose tissue and greater body adipose tissue mass compared with younger control subjects (5). The pattern of gene expression that occurs during excessive accumulation of subcutaneous and visceral adipose tissues, as detected in the present study, may lay a useful foundation for exploration in preclinical trials and future investigation for human diseases.

Collectively, due to the small sample size in the present preliminary study, the results should be confirmed in larger cohorts. As stated in the Materials and methods, the selection criteria of obese rats were based on a previous study (18), where 2 months of feeding with twice the amount of the ordinary diet induced a significant increase visceral adipose tissue accumulation. The group sizes for this study $(n=3)$ were based on the principles of 3Rs (Replacement, Reduction and Refinement) for humane animal research. A small sample size reduces the significance level of the study and increases the margin of error, which can render the study meaningless, as well as leading to cases of bias. In addition, a small sample size may also prevent the findings from being extrapolated (36). However, the present study identifed the same results as our previous study that was conducted using the same strategy, thus proving that the experiment is reproducible (18).

In conclusion, the expression of BRS3, UCP1 and PPARG were predicted to be associated with early accumulation of visceral adipose tissue in the body. However, careful validation must be applied before this gene network can be used for studies of visceral adipose tissue-associated diseases, including cancers, and the development of anti-disease agents.

\section{Acknowledgements}

The authors thank Professor Hiroshi Sugiyama and the team at Department of Chemistry, Graduate School of Science of Kyoto University, Kyoto for their technical support. The corresponding author would also like to thank Mr. Hiroshi Katsumoto, Thermo Fisher Scientific, Life Technologies, Tokyo, Japan for allowing the use of the Primer Express ${ }^{\mathrm{TM}}$ Software v3.0.1 License for primer design.

\section{Funding}

The present study was funded by a Fundamental Research Grant Scheme (grant no. 203/CIPPM/6711119) from the Ministry of Higher Education and partly funded by a Long-Term Research Grant Scheme-Malaysia Research University Network (grant no. 203/CIPPM/6720020). The second author was also supported by a USM fellowship from the Institute of Postgraduate Studies, Universiti Sains Malaysia.

\section{Availability of data and materials}

The datasets used and/or analyzed during the current study are available from the corresponding author on reasonable request.

\section{Authors' contributions}

JCY conceived the present study. BYK collected and analyzed data. TY and SKL performed experiments. BYK and GBYT designed the study. BYK and GBYT were responsible for 
supervising the study. BYK was responsible for visualization of data. TY wrote the original draft of the manuscript, and BYK reviewed and edited the manuscript.

\section{Ethics approval and consent to participate}

Experiments involving the use of animals were performed according to the Guidelines for the Care and Use of Animals for Scientific Purposes (19) after obtaining the ethical approval from the Universiti Sains Malaysia Animal Ethics Committee [permit no. USM/PPSF/50(085) Jld.2].

\section{Patient consent for publication}

Not applicable.

\section{Competing interests}

The authors declare that they have no competing interests.

\section{References}

1. Sharma AM and Staels B: Review: Peroxisome proliferator-activated receptor gamma and adipose tissueunderstanding obesity-related changes in regulation of lipid and glucose metabolism. J Clin Endocrinol Metab 92: 386-395, 2007.

2. Wajchenberg BL: Subcutaneous and visceral adipose tissue: Their relation to the metabolic syndrome. Endocr Rev 21: 697-738, 2000

3. Bjørndal B, Burri L, Staalesen V, Skorve J and Berge RK: Different adipose depots: Their role in the development of metabolic syndrome and mitochondrial response to hypolipidemic agents. J Obes 2011: 490650, 2011.

4. Shuster A, Patlas M, Pinthus JH and Mourtzakis M: The clinical importance of visceral adiposity: A critical review of methods for visceral adipose tissue analysis. Br J Radiol 85: 1-10, 2012.

5. Pascot A, Lemieux S, Lemieux I, Prud'homme D, Tremblay A, Bouchard C, Nadeau A, Couillard C, Tchernof A, Bergeron J and Després JP: Age-related increase in visceral adipose tissue and body fat and the metabolic risk profile of premenopausal women. Diabetes Care 22: 1471-1478, 1999.

6. Romieu I, Dossus L, Barquera S, Blottière HM, Franks PW, Gunter M, Hwalla N, Hursting SD, Leitzmann M, Margetts B, et al: Energy balance and obesity: What are the main drivers? Cancer Causes Control 28: 247-258, 2017.

7. Swinburn BA, Caterson I, Seidell JC and James WP: Diet, nutrition and the prevention of excess weight gain and obesity. Public Health Nutr 7: 123-146, 2004.

8. Fonseca DC, Sala P, Ferreira BAM, Reis J, Torrinhas RS, Bendavid I and Waitzberg DL: Body weight control and energy expenditure. Clin Nutr Exp 20: 55-59, 2018.

9. Parİ̃̂Âzkova J: Dietary habits and nutritional status in adolescents in central and eastern europe. Eur J Clin Nutr 54 (Suppl 1): S36-S40, 2000.

10. Racette SB, Deusinger SS and Deusinger RH: Obesity: Overview of prevalence, etiology, and treatment. Phys Ther 83: 276-288, 2003.

11. De Lorenzo A, Soldati L, Sarlo F, Calvani M, Di Lorenzo N and Di Renzo L: New obesity classification criteria as a tool for bariatric surgery indication. World J Gastroenterol 22: 681-703, 2016.

12. Cozzo AJ, Fuller AM and Makowski L: Contribution of adipose tissue to development of cancer. Compr Physiol 8: 237-282, 2017.

13. Li L, Wang G, Li N, Yu H, Si J and Wang J: Identification of key genes and pathways associated with obesity in children. Exp Ther Med 14: 1065-1073, 2017.

14. Choe SS, Huh JY, Hwang IJ, Kim JI and Kim JB: Adipose tissue remodeling: Its role in energy metabolism and metabolic disorders. Front Endocrinol (Lausanne) 7: 30, 2016.

15. Luo L and Liu M: Adipose tissue in control of metabolism. J Endocrinol 231: R77-R99, 2016.
16. Khoo BY, Najimudin N and Muhammad TS: The PPARgamma coding region and its role in visceral obesity. Biochem Biophys Res Commun 371: 177-179, 2008.

17. Longo M, Zatterale F, Naderi J, Parrillo L, Formisano P, Raciti GA, Beguinot F and Miele C: Adipose tissue dysfunction as determinant of obesity-associated metabolic complications. Int J Mol Sci 20: E2358, 2019.

18. Yogarajah T, Bee YT, Noordin R and Yin KB: Increased peroxisome proliferator-activated receptor $\gamma$ expression levels in visceral adipose tissue, and serum CCL2 and interleukin-6 levels during visceral adipose tissue accumulation. Mol Med Rep 11: 515-520, 2015.

19. Division of Research and Innovation: Guidelines for the care and use of animals for scientific purposes. Universiti Sains Malaysia, $2016 \mathrm{http} / / / \mathrm{www} . r e s e a r c h . u s m . m y / d e f a u l t . a s p ? t a g=10 \& \mathrm{f}=1 \& \mathrm{k}=1$.

20. Livak KJ and Schmittgen TD: Analysis of relative gene expression data using real-time quantitative PCR and the 2(-Delta Delta C(T)) method. Methods 25: 402-408, 2001.

21. Ashburner M, Ball CA, Blake JA, Botstein D, Butler $\mathrm{H}$, Cherry JM, Davis AP, Dolinski K, Dwight SS, Eppig JT, et al: Gene ontology: Tool for the unification of biology. The Gene Ontology Consortium. Nat Genet 25: 25-29, 2000.

22. The Gene Ontology Consortium: The gene ontology resource: 20 years and still GOing strong. Nucleic Acids Res 47: D330-D338, 2019.

23. Mi H, Muruganujan A, Ebert D, Huang $X$ and Thomas PD: PANTHER version 14: More genomes, a new PANTHER GO-slim and improvements in enrichment analysis tools. Nucleic Acids Res 47: D419-D426, 2019.

24. Nadarajan K, Balaram P and Khoo BY: MK886 inhibits the pioglitazone-induced anti-invasion of MDA-MB-231 cells is associated with PPAR $\alpha / \gamma$, FGF4 and 5LOX. Cytotechnology 68: 1771-1787, 2016.

25. Desvergne B and Wahli W: Peroxisome proliferator-activated receptors: Nuclear control of metabolism. Endocr Rev 20: 649-688, 1999.

26. Yang WL and Frucht $H$ : Activation of the PPAR pathway induces apoptosis and COX-2 inhibition in HT-29 human colon cancer cells. Carcinogenesis 22: 1379-1383, 2001.

27. Lee YH, Mottillo EP and Granneman JG: Adipose tissue plasticity from WAT to BAT and in between. Biochim Biophys Acta Mol Basis Dis 1842: 358-369, 2014.

28. Giralt M and Villarroya F: White, brown, beige/brite: Different adipose cells for different functions? Endocrinology 154: 2992-3000, 2013.

29. Park HS, Ju UI, Park JW, Song JY, Shin DH, Lee KH, Jeong LS, Yu J, Lee HW, Cho JY, et al: PPAR $\gamma$ neddylation essential for adipogenesis is a potential target for treating obesity. Cell Death Differ 23: 1296-1311, 2016.

30. Ghaben AL and Scherer PE: Adipogenesis and metabolic health. Nature Reviews Molecular Cell Biology volume 20: 242-258, 2019.

31. Lateef DM, Xiao C, Brychta RJ, Diedrich A, Schnermann J and Reitman ML: Bombesin-like receptor 3 regulates blood pressure and heart rate via a central sympathetic mechanism. Am J Physiol Heart Circ Physiol 310: H891-H898, 2016.

32. Zheng Q, Lin J, Huang J, Zhang H, Zhang R, Zhang X, Cao C, Hambly C, Qin G, Yao J, et al: Reconstitution of UCP1 using CRISPR/Cas9 in the white adipose tissue of pigs decreases fat deposition and improves thermogenic capacity. Proc Natl Acad Sci USA 114: E9474-E9482, 2017.

33. Villarroya F, Iglesias R and Giralt M: PPARs in the control of uncoupling proteins gene expression. PPAR Res 2007: 74364, 2007.

34. Yamada K, Ohki-Hamazaki H and Wada K: Differential effects of social isolation upon body weight, food consumption, and responsiveness to novel and social environment in bombesin receptor subtype-3 (BRS-3) deficient mice. Physiol Behav 68: 555-561, 2000.

35. Blücher C and Stadler SC: Obesity and breast cancer: Current insights on the role of fatty acids and lipid metabolism in promoting breast cancer growth and progression. Front Endocrinol (Lausanne) 8: 293, 2017.

36. Faber $\mathrm{J}$ and Fonseca LM: How sample size influences research outcomes. Dental Press J Orthod 19: 27-29, 2014.

This work is licensed under a Creative Commons Attribution-NonCommercial-NoDerivatives 4.0 International (CC BY-NC-ND 4.0) License. 